\title{
Automatización de actos de devolución en el software Cabri LM ${ }^{1}$
}

\author{
Automating events back in the LM Cabri \\ Automatizando eventos de volta na LM Cabri
}

Recibido: mayo de 2013

Aceptado: agosto de 2013
Marisol Rueda Puentes ${ }^{2}$ Ángel Miguel Niño Navas ${ }^{3}$

Martín Acosta Gempeler ${ }^{4}$

\section{Resumen}

Ésta es una investigación de tipo ingeniería didáctica, en la cual se adoptó actividades de matemática recreativa por medio del software Cabri LM, intentando automatizar los actos de devolución abordados por Guy Brousseau en su Teoría de las Situaciones Didácticas, que debe tener en cuenta el docente en las intervenciones durante la clase como una reacción a una acción.

Palabras clave: Teoría de las situaciones didácticas; aula; recursos didácticos; recursos informáticos; software dinámico; automatización de actos de devolución; análisis didáctico.

\section{Abstract}

This is a didactic engineering type research, which was adopted recreational mathematics activities through LM Cabri, trying to automate back acts addressed by Guy Brousseau's Theory of didactic situations, you should take into account the teacher interventions during class as a reaction to an action.

Keywords: Theory of didactic situations, classroom teaching resources, computing resources, dynamic software, automating back actions, training analysis.

\section{Resumo}

Esta é uma pesquisa do tipo engenharia didática, que foi aprovada atividades recreativas matemática através LM Cabri, tentando automatizar volta atos abordados pela Teoria das situações didáticas de Guy Brousseau, você deve levar em conta o professor intervenções durante a aula como uma reação a uma ação.

Palavras-chave: Teoria das situações didáticas, recursos didáticos em sala de aula, os recursos de computação, software dinâmico, automatização de volta ações, análise de formação.

1 Articulo de Investigación

2 Universidad Industrial de Santander. Grupo Edumat. Colombia. Contacto: mruedapu@matematicas.uis.edu.co

3 Universidad Industrial de Santander. Grupo Edumat. Colombia. Contacto: alfarzan77@hotmail.com

4 Universidad Industrial de Santander. Grupo Edumat. Colombia. Contacto: maedu@hotmail.com 


\section{Introducción}

Las investigaciones pedagógicas muestran que los alumnos aprenden y retienen mejor lo que tocan y manipulan. Por eso el software interactivo tiene un impacto positivo en el aprendizaje porque permite a los alumnos manipular en la pantalla del computador objetos que corresponden a conceptos teóricos, pero que al mismo tiempo adquieren una realidad perceptible. De esta manera, el software se convierte en un puente entre el mundo real que rodea al alumno y el mundo abstracto de las matemáticas.

Por otra parte, según los planteamientos de la matemática recreativa, uno de los factores que incide positivamente en el aprendizaje es el modo de intervención del profesor, que busca una mayor implicación de los estudiantes fomentando la identificación de errores, la búsqueda de comprensión y la comunicación de procesos. Estas intervenciones son el fruto de los actos de devolución que realiza el docente.

En esta investigación pretendemos automatizar esos actos de devolución en el software Cabri LM de manera que los estudiantes reciban la retroalimentación que buscan por medio de la identificación de sus errores y la comprensión de sus procesos.

Teniendo en cuenta lo anterior, podemos formular el problema de la siguiente manera: ¿Cómo utilizar Cabri LM para automatizar actos de devolución en actividades de matemática recreativa y calendario matemático, de manera que se favorezca el empoderamiento matemático de estudiantes de básica primaria entre los 10 y 11 años?

\section{Referentes teóricos}

Se utilizo la teoría de las situaciones didácticas para analizar las actividades de Matemática Recreativa y Semillero Matemático con el fin de identificar las decisiones didácticas. Los conceptos clave son los de devolución y validación, según los cuales el estudiante asume la responsabilidad de resolver una situación problema y está en capacidad de decidir si el resultado es correcto; de lo contrario, debe corregirlo.

Por otra parte, la teoría de las situaciones didácticas dará argumentos para automatizar las decisiones didácticas a través del software CABRI LM. El proceso (acto) de devolución se refiere a la forma como el profesor interviene en la interacción del estudiante con el medio, con el fin de garantizar la posibilidad de la validación. Al combinar las actividades de Matemática Recreativa con el software Cabri LM y aplicando la TSD se piensa que se debe obtendrá una nueva herramienta que mejore la enseñanza de las Matemáticas.

\section{Metodología}

Se utilizó la metodología de ingeniería didáctica, que comprende tres fases: diseño, experimentación y validación.

Población. Se trabajó con un grupo de estudiantes de básica primaria entre los 10 y 11 años.

Toma de datos. Registro en vídeo de tres parejas de estudiantes y notas de campo de los observadores.

\section{Análisis de datos y conclusiones}

Consideramos este trabajo como un primer esfuerzo de automatizar actos de devolución en actividades de matemática recreativa, que necesariamente tiene limitaciones, pero que abrió perspectivas interesantes para el desarrollo de la educación matemática y el uso de las tecnologías computacionales. El software Cabri LM, al posibilitar la representación informática del trabajo del estudiante y la programación flexible de las retroacciones, genera oportunidades de automatizar acciones que antes eran exclusivas del profesor. Esta nueva situación requiere un estudio cuidadoso para determinar las 
ventajas y desventajas para el aprendizaje de los estudiantes. Nuestra experimentación nos permite prever un efecto positivo de la automatización de los actos de devolución, pero es necesario hacer estudios con un mayor número de estudiantes

\section{Referencias}

De Guzmán, Miguel. (1984). Juegos matemáticos en la enseñanza. En Actas de las IV JAEM. Tenerife (pp. 49-85)
Margolinas, C. (2009) La importancia de lo verdadero y lo falso en la clase de matemáticas. (Martín Eduardo Acosta \& Jorge Fiallo, trad.) Colombia: Universidad Industrial de Santander, UIS. (Obra original publicada en 1998)

Mora, A. \& Lucena, A. (2008) Conceptualización de la traslación con la mediación del programa Cabri LM. Trabajo de grado. Escuela de matemáticas. Universidad Industrial de Santander. 